\title{
Consensus on Training and Implementation of Enhanced Recovery After Surgery: A Delphi Study
}

\author{
Nader K. Francis ${ }^{1,2}$ - Thomas Walker ${ }^{1}$ - Fiona Carter ${ }^{3}$ - Martin Hübner ${ }^{4}$. \\ Angela Balfour ${ }^{5}$ - Dorthe Hjort Jakobsen ${ }^{6} \cdot$ Jennie Burch $^{7}$ • Tracy Wasylak ${ }^{8,9}$. \\ Nicolas Demartines ${ }^{4} \cdot$ Dileep N. Lobo $^{10} \cdot$ Valerie Addor $^{4} \cdot$ Olle Ljungqvist $^{11}$
}

Published online: 4 January 2018

(C) Société Internationale de Chirurgie 2018

\begin{abstract}
Background Enhanced Recovery After Surgery (ERAS) is widely accepted in current surgical practice due to its positive impact on patient outcomes. The successful implementation of ERAS is challenging and compliance with protocols varies widely. Continual staff education is essential for successful ERAS programmes. Teaching modalities exist, but there remains no agreement regarding the optimal training curriculum or how its effectiveness is assessed. We aimed to draw consensus from an expert panel regarding the successful training and implementation of ERAS. Methods A modified Delphi technique was used; three rounds of questionnaires were sent to 58 selected international experts from 11 countries across multiple ERAS specialities and multidisciplinary teams (MDT) between January 2016 and February 2017. We interrogated opinion regarding four topics: (1) the components of a training curriculum and the structure of training courses; (2) the optimal framework for successful implementation and audit of ERAS including a guide for data collection; (3) a framework to assess the effectiveness of training; (4) criteria to define ERAS training centres of excellence.

Results An ERAS training course must cover the evidence-based principles of ERAS with team-oriented training. Successful implementation requires strong leadership, an ERAS facilitator and an effective MDT. Effectiveness of training can be measured by improved compliance. A training centre of excellence should show a willingness to teach and demonstrable team working.

Conclusions We propose an international expert consensus providing an ERAS training curriculum, a framework for successful implementation, methods for assessing effectiveness of training and a definition of ERAS training centres of excellence.
\end{abstract}

The data in this paper have, in part, been presented at the following meeting: The international ERAS Conference: Lyon May 2017.

Nader K. Francis

nader.francis@ydh.nhs.uk

1 Department of Colorectal Surgery, Yeovil District Hospital Foundation Trust, Higher Kingston, Yeovil, Somerset BA21 4AT, UK

2 Faculty of Science, University of Bath, Wessex House 3.22, Bath BA2 7AY, UK

3 South West Surgical Training Network, ERAS-UK, Yeovil, Somerset BA20 2RH, UK
4 Department of Visceral Surgery, Lausanne University Hospital (CHUV), Rue de Bugnon 46, 1011 Lausanne, Switzerland

5 NHS Lothian Western General Hospital, Crewe Road, Edinburgh EH4 2XU, Scotland

6 Section of Surgical Pathophysiology 4074, Rigshospitalet, Blegdamsvej 9, 2100 Kbh Ø, Copenhagen, Denmark

7 Head of Gastrointestinal Nurse Education, Academic Institute, St Mark's Hospital, London HA1 3UJ, UK 


\section{Introduction}

Enhanced Recovery After Surgery (ERAS) protocols aim to reduce the stress response to surgery and optimise postoperative recovery by employing a multimodal approach to perioperative management. The safety of ERAS protocols have been demonstrated in several randomised trials [1, 2], and numerous studies and meta-analyses have shown the efficacy of ERAS in colorectal surgery [3-5]. Improved outcomes have now been demonstrated across multiple surgical specialities including benign and malignant upper gastrointestinal surgery, hepato-pancreato-biliary surgery, thoracic surgery, urology, gynaecology and orthopaedic surgery [6].

It has been shown that improved compliance with ERAS protocols results in better short-term outcomes in terms of length of stay, re-admission and complications [3]. Difficulties in protocol implementation and compliance, maintenance of protocols and patient, staff, practice and/or resource factors have all been implicated as reasons why the success by the pioneering groups has been hard to replicate [7, 8]. The post-operative elements of ERAS protocols are especially difficult to achieve high levels of compliance [7, 9].

Maintaining a successful ERAS programme requires continual staff education across the whole multidisciplinary team MDT [10]. There are a multitude of training resources currently available with the aim of promoting good practice in ERAS. However, despite these resources, there is no current consensus on the optimum content (curriculum) for ERAS training or the best method to deliver this training. There is also a need for a framework to assess effectiveness of training and education in ERAS and criteria to define expert sites in ERAS training-centres of excellence.

The aim of this study was to draw consensus from an expert panel on the key elements of an ERAS training curriculum and how such a curriculum should be delivered, key factors for successful implementation of ERAS, the

8 Strategic Clinical Networks, Alberta Health Services, Edmonton, AB, Canada

9 Faculty of Nursing, University of Calgary, 10301 Southport Lane SW, Calgary, AB T2W1S7, Canada

10 Gastrointestinal Surgery, Nottingham Digestive Diseases Centre, National Institute for Health Research (NIHR) Nottingham Biomedical Research Centre, Nottingham University Hospitals NHS Trust and University of Nottingham, Queen's Medical Centre, Nottingham NG7 2UH, UK

11 Department of Surgery, Faculty of Medicine and Health, Örebro University, 70185 Orebro, Sweden optimal method of assessment of ERAS training and the criteria to identify centres of excellence in ERAS training.

\section{Materials and methods}

An initial focus group was formed among the authorship (NKF, MH, AB, DHJ, JB, TW, ND, DL and OL), and four topics within the theme of training and implementation of ERAS were proposed as areas in need of further interrogation:

1. The components of a training curriculum and the structure of training courses.

2. The optimal framework for successful implementation and audit of ERAS including a guide for data collection.

3. A framework to assess the effectiveness of ERAS training.

4. Criteria to define ERAS training centres of excellence.

\section{Delphi process}

A modified Delphi technique was used to gain consensus on these four topics. This involves a reiterative process of interrogation of a group of experts. Expert opinion from various sources is combined using qualitative then quantitative methods with the aim of converging on a shared consensus result $[11,12]$. Notable characteristics of the Delphi technique include: anonymity, controlled feedback of opinion, reiteration of concept and potential for application of statistical analysis techniques. It has been widely used in healthcare in areas such as epidemiology, education and improving clinical practice [13-15].

This modified Delphi study involved three rounds of questioning to an expert group between January 2016 and February 2017 and an interactive focus group of ERAS novices at the international ERAS conference in Lisbon on 27-30 April 2016.

Fifty-eight experts were chosen to complete the first round questionnaire. Experts were selected based on their peer standing as pioneers or early adopters of ERAS and from multiple specialties including colorectal surgery, urology, gynaecology, upper gastrointestinal surgery, hepatobiliary surgery, orthopaedic surgery and elderly care (Fig. 1). The experts included representation from a wide range of healthcare professionals including surgeons, anaesthetists, nurses, educationalists, healthcare management and patient public representatives (Fig. 2) and came from a number of countries including the UK, Denmark, Sweden, Switzerland, France, Germany, the Netherlands, Spain, Canada, USA and New Zealand. 
Fig. 1 Speciality of selected expert panel

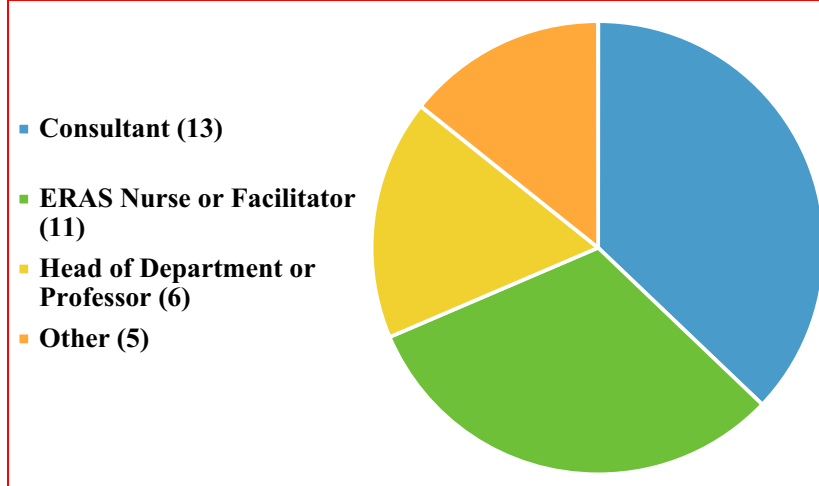

Fig. 2 Positions held by members of selected expert panel

Open questions were formulated and experts were invited by e-mail to complete an online questionnaire using Survey Monkey or to complete a paper version and return by post. Non-responders received two reminders by e-mail. The responses to this first round were then collated to generate a limited number of statements or choices to form the second round questionnaire. In the second round, experts were then asked whether they agreed or disagreed with each statement presented in the questionnaire.

\section{Focus group}

The outcome of the second round was presented to a focus group (12 people) at the international ERAS conference in Lisbon on 27-30 April 2016. The focus group was facilitated by three expert authors (NKF, MH and DL), and all delegates at the conference were invited to attend regardless of level of experience with training or implementation of ERAS. The focus group aimed to contextualise the experts' opinions within the reality of different health care systems in order to improve the generalisability of the consensus statements. The opinions expressed by those in the group were considered in the formulation and modification of the questions for round three of interrogation. The qualitative phase data following the focus group was transcribed and analysed by FC and added to the structure and format of the final rounds questionnaire. This included editing or adding new questions prior to final vote.

The final round aimed to obtain a final consensus. An online survey was designed and was sent out to the experts in January 2017, and the experts were asked to indicate their level of agreement to a set of statements or questions based on a Likert scale from 1 to 5 ( $1=$ strongly disagree, $5=$ strongly agree) or select applicable answers (from suggestions based on the workshop and first two rounds of voting). Participants were also invited to make any additional suggestions or comments.

\section{Data analysis}

This was based on percentage response rates and the consensus data was reported as the weighted average score for each question. Any additional questions in the final round were presented as percentages. A pragmatic approach was applied by the study review panel, whereby top ranked responses to each question over the 70th percentile were deemed to have met consensus.

\section{Results}

Thirty-five experts responded to the first round questionnaire $(60.34 \%)$. Thirty-three $(56.9 \%)$ experts responded to the second round, and $31(53.4 \%)$ to the final round. 
Of the experts completing the first round questionnaire, $25(71 \%)$ had over 5 years of experience utilising ERAS protocols or principles (Fig. 3), and 21 (60\%) had over 5 years of ERAS teaching experience (Fig. 4). Fifteen experts included in this consensus $(26 \%)$ have been influential in implementing ERAS programmes in over 150 sites (Fig. 5).

Following presentation of the second round at the focus group in Lisbon, seven further questions were added to the final round questionnaire that were felt to be of additional value (Fig. 6).

Several elements were also added to existing elements of the questionnaire: regarding MDT implementation,

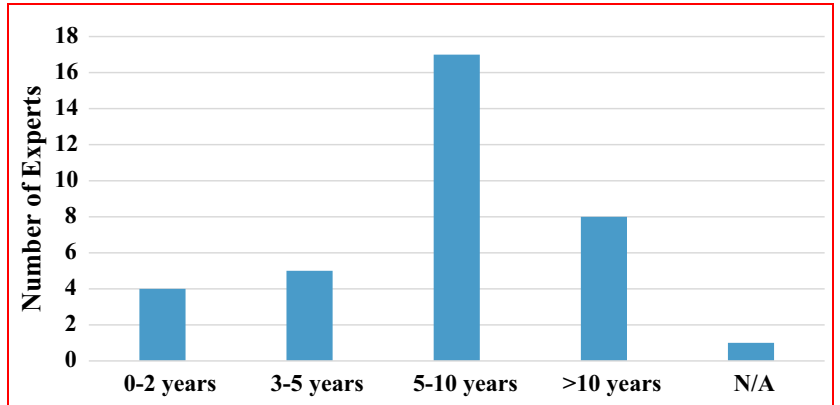

Fig. 3 Length of time utilising ERAS protocols by selected expert panel

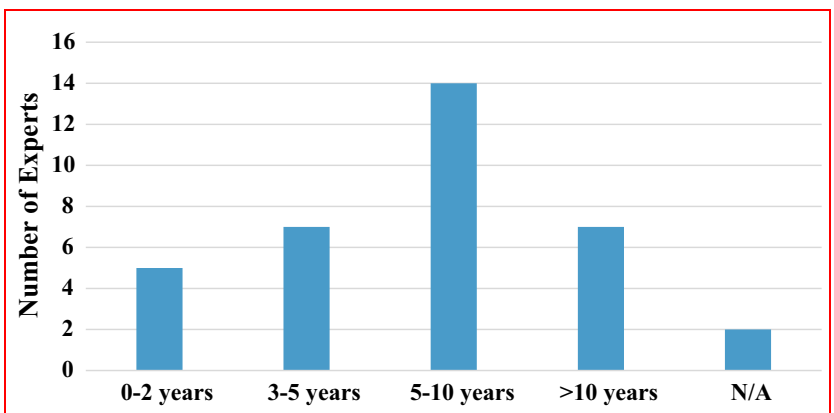

Fig. 4 Length of time teaching ERAS by selected expert panel

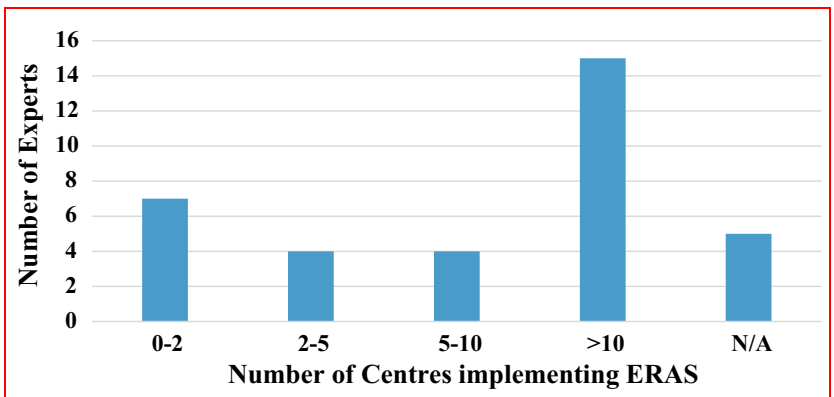

Fig. 5 Number of centres implementing ERAS following training delivered by selected experts
"Awareness of ERAS among the team" was added to questioning; regarding Data collection questioning, "Patient-reported outcome measures", "readiness of discharge" and "complications" was added; regarding defining ERAS expert sites, "ability to adapt to local challenges" was added to questioning.

Following the final round, expert panel consensus statements were made in all four domains of ERAS training and implementation interrogation: (1) the components of a training curriculum and the structure of training courses; (2) the optimal framework for successful implementation and audit of ERAS including a guide for data collection; (3) a framework to assess the effectiveness of ERAS training; (4) criteria to define ERAS training centres of excellence.

\section{The components of a training curriculum and the structure of training courses}

Expert consensus was achieved regarding the elements that should be included within an ERAS training curriculum (Fig. 7). The most important components included teaching up-to-date evidence-based principles of ERAS, audit and feedback processes, and integrating the whole patient journey into training. A didactic teaching course with clinical components was the least desirable part of the curriculum.

It was agreed that teaching methods to deliver an ERAS curriculum should incorporate both individual and MDTbased learning methods. The expert panel suggested that the ERAS curriculum should be taught via team-based training with a multidisciplinary faculty (96.67\%), face-toface training $(93.33 \%)$, online modules/e-learning with integrated assessment $(76.67 \%)$ and as part of the undergraduate nursing and medical curricula (76.67\%). Continual audit and feedback should be performed to demonstrate the benefits of teaching methods (73.33\%). Apart from educating healthcare professionals, patients should be informed about ERAS with pathway "walkthrough" methods $(70 \%)$.

\section{The optimal framework for successful implementation and audit of ERAS including a guide for data collection}

Consensus was reached regarding the essential elements for optimum ERAS implementation (Fig. 8). Highlighting the importance of MDT working, effective leadership and continual audit and data collection. Essential elements also included management support, a dedicated ERAS nurse or facilitator and promoting awareness of ERAS among the whole surgical team. 


\section{Multidisciplinary team working and learning together can be best acheived by...choose all that apply}

- Regular scheduled meetings

- Clearly aligned goals for patient care

- Collaborative learning sessions

- Feedback of results to whole team

- Strong leadership and vision

- Collaborative development of pathways

Which of the following attributes are important for "leadership by a senior clinician"?...choose all that apply

- Leader must be positive and passionate about ERAS

- ERAS lead must be locally respected and able to influence clinical and management colleagues

- Identify an early adopter of ERAS locally

- Create a small stipend or funded sessions to lead ERAS

- Provide project management support

- ERAS lead should be a surgeon or anaesthetist

- ERAS lead could be from any relevant role in the team

How can "management support" be acheived?...choose all that apply

- Engagement with senior management

- Incentives through commissioning or funding

- Include senior manager in ERAS team

- Share positive outcomes with senior management

- Link clinical outcomes to organisational objectives

Which of the following are useful to secure the "involvement of an ERAS nurse or facilitator?...choose all that apply

- Demonstrate a clear financial incentive to create and fund the role

- Current staff member may "self select" and adapt their job description

- Clarify the role and responsibilities

- More than one facilitator is required to ensure continuity

- Enthusiastic team member, with the correct skills and abilities, should have funded time available to develop the role

How can the success of an expert centre's own ERAS programme be practically demonstrated?...choose all that apply

- Publication of data on specific criteria

- Using Encare database/reporting too

- Presence of an ERAS nurse or facilitator

- Peer review

- Centre visit and observation

- Team scorecard or survey

- Summary reports of training sessions, including feedback

- Attending and presenting at regional, national or international meetings

The ERAS curriculum should be taught using the following methods...choose all that apply

- Online modules/e-learning with assessment

- Face to face training

- Modular, self directed learning

- Expert email follow up for ongoing queries

- Team based training with a multidisciplinary faculty

- Cased based discussion

- Patient experiences and pathway "walkthrough", involving patients in the course

- ERAS audit system

- Didactic teaching to cover the fundamental and evidence of ERAS

- Include more than one set of learniners in the course

- Training should be bespoke for each institution

- During staff induction

- As part of the undergraduate nursing and medical curricula

- Ward based learning, led by an ERAS nurse

- Ongoing audit and feedback to demonstrate the benefits

- Courses for different specialties should have a faculty lead from the same specialty

- Review the guidelines

The ERAS training course should be practically assessed using the following methods...choose all that apply

- Prompt feedback from participants after each course

- Online feedback forms

- Compulsary submission of data (from learners)

- Regular reporting to expert site

- Follow-up by faculty

Fig. 6 Questions added to round three of interrogation following workshop 
Fig. 7 ERAS training curriculum and course content

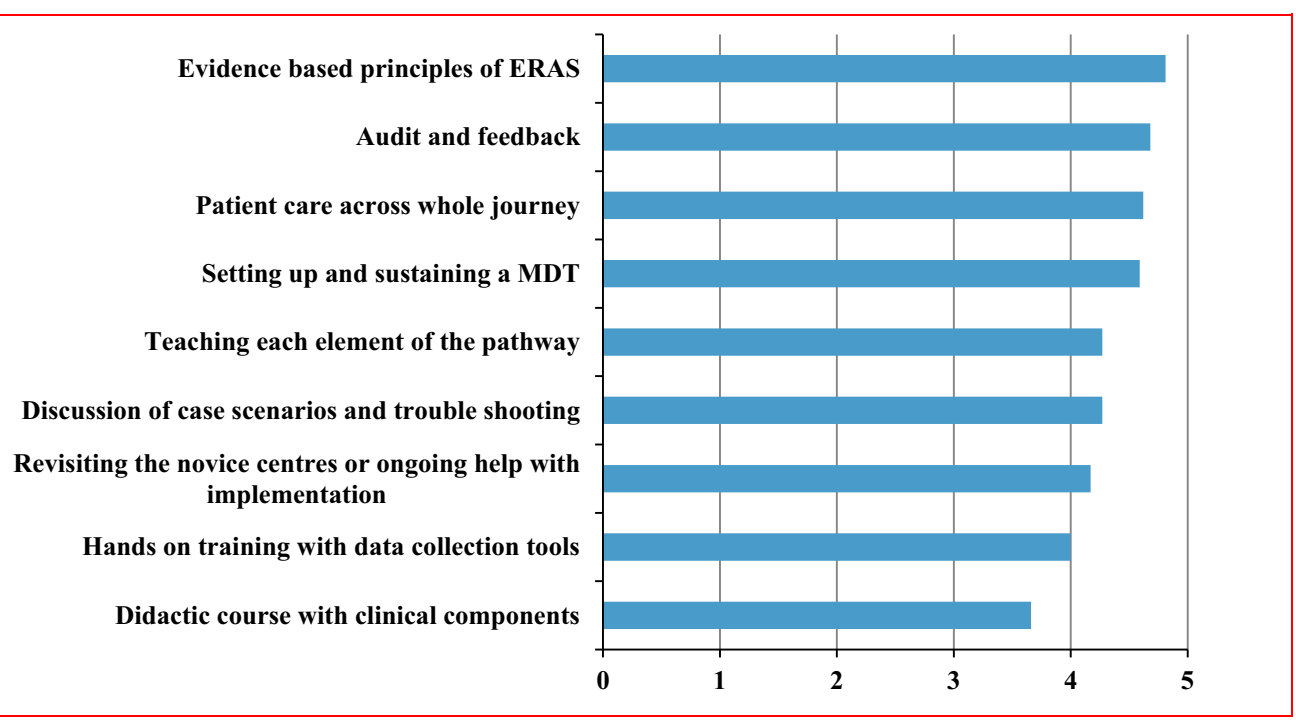

Fig. 8 Essential elements for optimum ERAS implementation Multidisciplinary team (MDT) working and
learning together

Leadership from a senior clinician Audit and data collection

Management support

ERAS nurse/ facilitator

Awareness of ERAS among the team

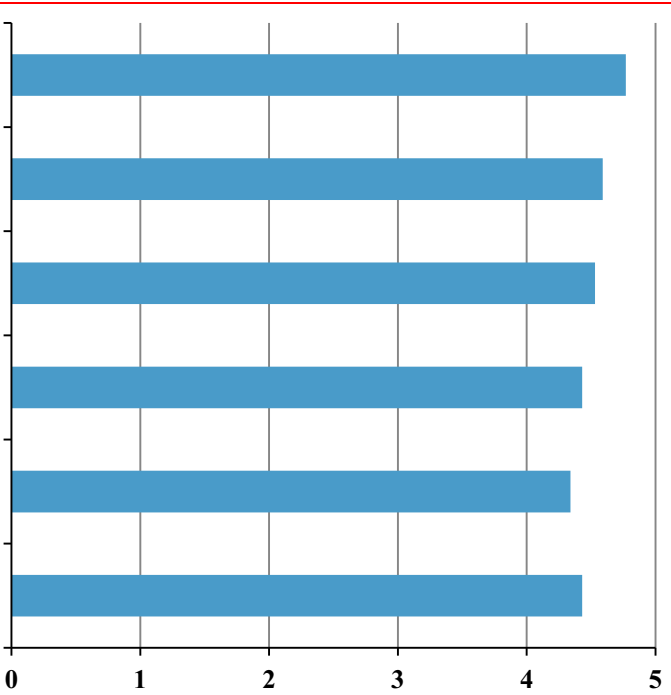

The experts suggested that MDT team working and learning together can be best achieved by development of an effective system of feedback (97\%), strong leadership and vision (90\%), regular scheduled meetings (80\%), clearly aligned goals for patient care $(80 \%)$ and collaborative development of ERAS pathways (77\%).

An agreement was also reached on the key characteristics of successful ERAS leaders. They must be passionate and positive about ERAS (97\%), locally respected and able to influence clinical and management colleagues (97\%).

Senior management engagement was deemed by the experts to be essential for ERAS success (87\%), as well as linking clinical outcomes of ERAS to organisational objectives (87\%), including a senior manager within the ERAS team (80\%) and sharing the positive outcomes of ERAS with senior management $(77 \%)$.
A dedicated ERAS nurse or facilitator was proposed by the experts to ensure optimum ERAS implementation. The ERAS facilitator, a role in the UK performed by nonclinical, managerial or administrative staff, should be enthusiastic team members and have the correct skills and abilities as well as funded time available to develop the role $(86.67 \%)$. They must also have clear roles and responsibilities $(83.33 \%)$.

The experts voted for what should constitute the minimum amount of ERAS data collected during an audit process (Fig. 9). They also agreed that the challenges of ERAS data collection include a lack of human resources (4.13), a lack of time (4.10) and non-automated or inefficient processes (4.00). Figure 10 outlines the methods the experts suggested to support collection of data relevant to the audit process. 


\section{A framework to assess the effectiveness of ERAS training}

The experts have proposed a framework by which the effectiveness of ERAS training can be assessed. This includes registering prompt feedback from course delegates and monitoring post-training compliance with ERAS components (Fig. 11).

Practically, assessment methods for ERAS teaching courses should include prompt feedback from participants after each course (96.67\%) and the follow-up of "trained" centres using faculty feedback (76.67\%). The feedback provided by faculty should be performed using standardised online feedback forms $(70.00 \%)$.

\section{Criteria to define ERAS training centres of excellence}

The expert panel agreed that a successful ERAS training centre should demonstrate: regular audit and data collection, a willingness to teach, effective teamwork, high compliance with ERAS elements and be adaptable to local implementation challenges (Fig. 12).

The success of an expert centre's own ERAS programme should be demonstrated by publication of data $(87.10 \%)$ together with scheduled observation visits (77.42\%) and both attendance and presentation at national or international meetings $(74.19 \%)$.

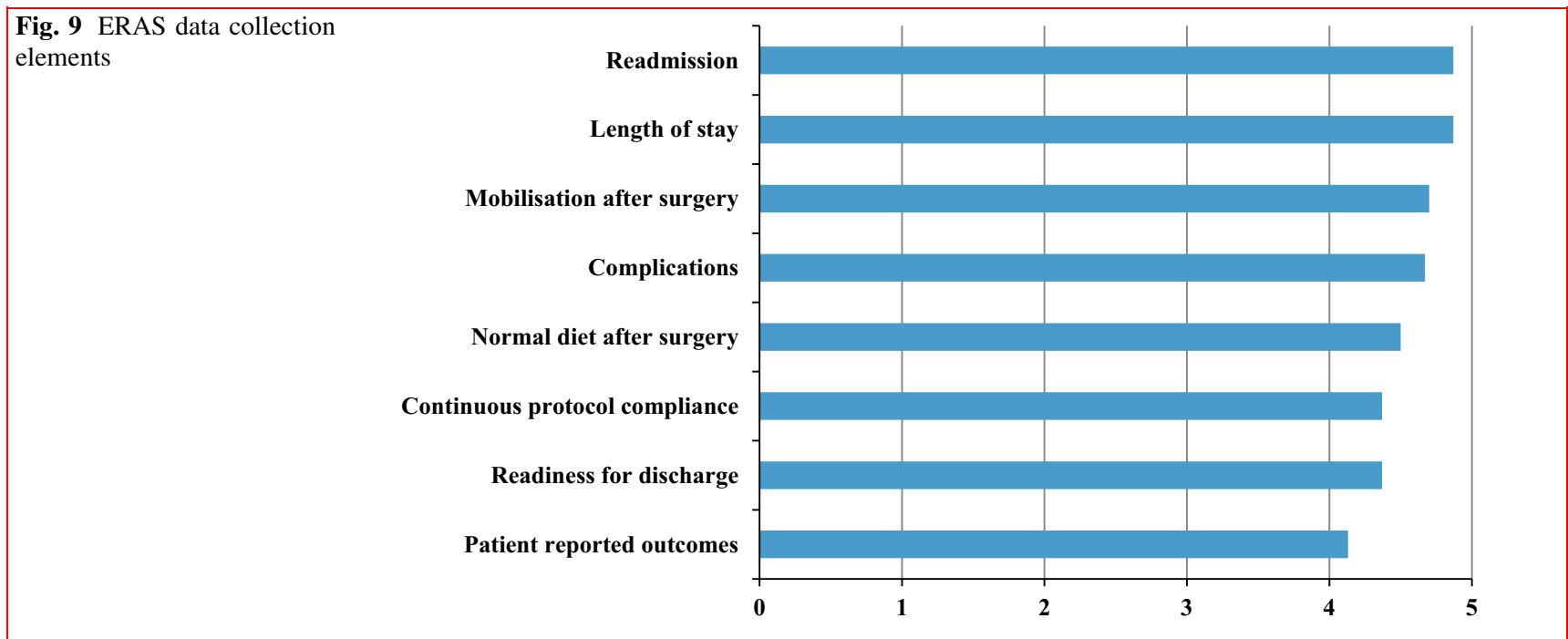

Fig. 10 Methods to support ERAS data collection

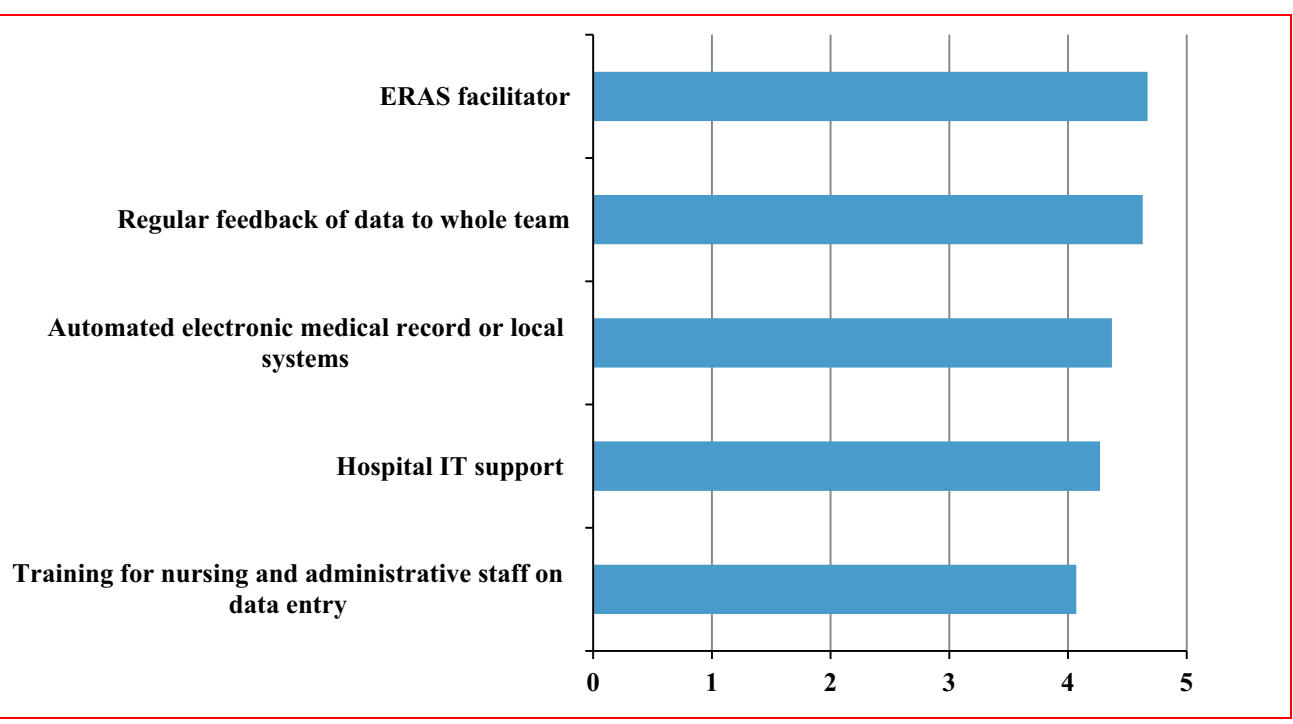


Fig. 11 Framework of assessment of training and education
Regular and prompt feedback from course
participants

Interdisciplinary teaching and learning

Post training compliance and outcomes

Interactive audit system
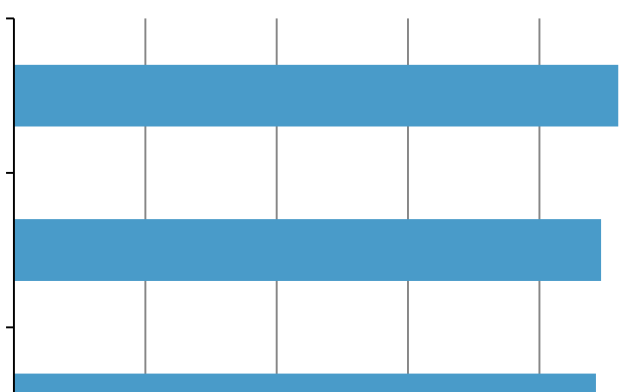

(
Fig. 12 Criteria to define expert ERAS training Centre

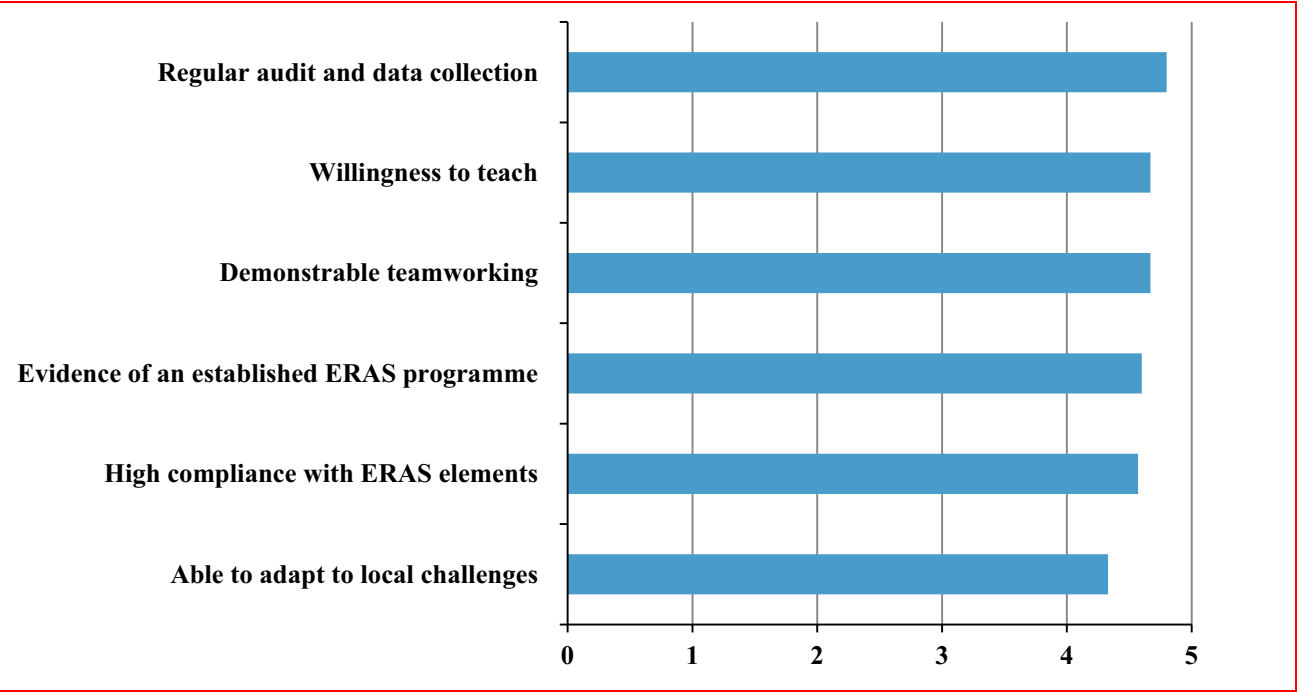

\section{Discussion}

Successful implementation of ERAS programmes requires continual staff education. Despite there being a large number of courses and implementation programmes available, there is no consensus on the essential elements required for successful training and implementation of ERAS.

To our knowledge, this is the first study to provide an agreed consensus on the content of a training curriculum, and a guide for implementation, training and audit of all aspects of ERAS. The proposed curriculum encompasses clear guidance on the optimal implementation and audit of ERAS including optimal training methods, methods of assessment and quality assurance of ERAS training, data collection and criteria for defining expert training centres.
A modified Delphi method was applied to identify and rank the most important elements of the training curriculum of ERAS and its implementation from an expert panel. It was also important to obtain novices' views on the issue of implementation, and hence we include a focus group into the design of this study. This final consensus document has summarised the essential components of ERAS training. Team-based training with a multidisciplinary team (MDT) approach has been recommended as the optimal method to deliver an evidence-based training curriculum.

Successful implementation requires strong leadership, a dedicated ERAS facilitator as well as an effective ERAS MDT. Having dedicated ERAS personnel with clearly defined roles was deemed to be essential for both data collection and feeding back the outcomes of ERAS to the MDT. The experts agreed on a minimum dataset for ERAS 
audit including clinical and functional outcomes in addition to protocol compliance.

Assessment of ERAS training has never been explored prior to this study. Collecting standardised feedback from both delegates and faculty, inter-disciplinary teaching and learning and monitoring ERAS compliance following training have been proposed by the expert panel as the best method to assess the real impact of ERAS training.

Willingness of a unit to teach and demonstrate effective team working is considered by our panel to be the top requirements to define ERAS expert centres. These centres should also show regular data collection and audit processes and sustain high compliance with ERAS elements. There is no doubt that the success of an ERAS centre relies on effective leadership. This Delphi process has now defined the criteria for identification of such a leader: they must be passionate and positive about ERAS and engage and include senior management in order to impact and sustain change.

Prior research on ERAS has focused mainly on the evidence supporting each element across different surgical specialities and recommended the role of clinical champions and the presence of a dedicated ERAS facilitator [16-19]. However, these guidelines did not address the practicalities of training or how to tackle difficulties with implementation. Through this study, we have been able to draw consensus regarding the essential components of a training curriculum, how that training is best delivered, how training quality should be assessed and how to identify those centres that are excelling in ERAS training delivery. We have also suggested practical methods for assessing how successful ERAS programmes could be demonstrated including publication of data on specific criteria, observation of successful centres and representation of success at national and international meetings.

There are a number of limitations to this study. Consensus statements in general are considered weak evidence. However, they are still the optimal method in identifying areas to channel further research and future practice, particularly when there are implementation challenges reflected by a wide variation in implementation and outcome as in ERAS [8]. The initial rounds of this study identified many local barriers and challenges to adopting and sustaining ERAS, and practical solutions were proposed in the final consensus statements. The selection of experts is another critical issue with consensus statements. The group of experts involved in this research were all recommended by their peers as experts in the field and they are generally distributed across Europe and beyond. The experts represented a broad range of different specialities and disciplines to ensure generalisability of the results. The experts have significant experience in developing and utilising ERAS protocols, extensive teaching experience and proven records in implementation of ERAS protocols following the delivery of training. In this study, the response rate was relatively high (over 60\%) compared to other similar studies, indicating the commitments of the experts to draw consensus on the given issues. Additionally, the contribution of the review panel of data collection could arguably dilute or alter the original intent. Although the review panel contributed to reducing redundancy, combining and clarifying the questions which are requirements of the Delphi process, the review panel made every effort in preserving the initial questions' intent.

In conclusion, a detailed framework for a structured ERAS training curriculum and implementation strategy has been proposed to ensure successful and sustained application of ERAS. The framework encompasses various training modalities and assessment and has the potential to provide quality control and potentiate future ERAS research to improve implementation and patient outcomes.

Acknowledgements The authors are grateful to the experts who contributed to this project.

\section{References}

1. Ren L, Zhu D, Wei Y et al (2012) Enhanced Recovery After Surgery (ERAS) program attenuates stress and accelerates recovery in patients after radical resection for colorectal cancer: a prospective randomized controlled trial. World J Surg 36(2):407-414. https://doi.org/10.1007/s00268-011-1348-4

2. Varadhan KK, Neal KR, Dejong $\mathrm{CH}$, Fearon KC, Ljungqvist $\mathrm{O}$, Lobo DN (2010) The enhanced recovery after surgery (ERAS) pathway for patients undergoing major elective open colorectal surgery: a meta-analysis of randomized controlled trials. Clin Nutr 29(4):434-440

3. Gustafsson UO, Hausel J, Thorell A et al (2011) Adherence to the enhanced recovery after surgery protocol and outcomes after colorectal cancer surgery. Arch Surg 146(5):571-577

4. Group EC (2015) The impact of enhanced recovery protocol compliance on elective colorectal cancer resection: results from an international registry. Ann Surg 261(6):1153-1159

5. Lv L, Shao YF, Zhou YB (2012) The enhanced recovery after surgery (ERAS) pathway for patients undergoing colorectal surgery: an update of meta-analysis of randomized controlled trials. Int J Colorectal Dis 27(12):1549-1554

6. Ljungqvist O, Scott M, Fearon KC (2017) Enhanced recovery after surgery: a review. JAMA Surg 152(3):292-298

7. Maessen J, Dejong CH, Hausel J et al (2007) A protocol is not enough to implement an enhanced recovery programme for colorectal resection. Br J Surg 94(2):224-231

8. Lyon A, Solomon MJ, Harrison JD (2014) A qualitative study assessing the barriers to implementation of enhanced recovery after surgery. World J Surg 38(6):1374-1380. https://doi.org/10. 1007/s00268-013-2441-7

9. Smart NJ, White P, Allison AS, Ockrim JB, Kennedy RH, Francis NK (2012) Deviation and failure of enhanced recovery after surgery following laparoscopic colorectal surgery: early prediction model. Colorectal Dis 14(10):e727-e734 
10. Keller DS, Delaney CP, Senagore AJ, Feldman LS, Force SST (2016) Uptake of enhanced recovery practices by SAGES members: a survey. Surg Endosc 31(9):3519-3526

11. Dalkey NC (1969) The Delphi method: An experimental study group of opinion. The RAND Corporation, Santa Monica

12. Williams PL, Webb C (1994) The Delphi technique: a methodological discussion. J Adv Nurs 19(1):180-186

13. Alahlafi A, Burge S (2005) What should undergraduate medical students know about psoriasis? Involving patients in curriculum development: modified Delphi technique. BMJ 330(7492):633-636

14. Gagliardi AR, Simunovic M, Langer B, Stern H, Brown AD (2005) Development of quality indicators for colorectal cancer surgery, using a 3-step modified Delphi approach. Can J Surg 48(6):441-452

15. Ferri CP, Prince M, Brayne C et al (2005) Global prevalence of dementia: a Delphi consensus study. Lancet 366(9503):2112-2117
16. Lassen K, Soop M, Nygren J et al (2009) Consensus review of optimal perioperative care in colorectal surgery: enhanced Recovery After Surgery (ERAS) Group recommendations. Arch Surg 144(10):961-969

17. Feldheiser A, Aziz O, Baldini G et al (2016) Enhanced Recovery After Surgery (ERAS) for gastrointestinal surgery, part 2: consensus statement for anaesthesia practice. Acta Anaesthesiol Scand 60(3):289-334

18. Scott MJ, Baldini G, Fearon KC et al (2015) Enhanced Recovery After Surgery (ERAS) for gastrointestinal surgery, part 1: pathophysiological considerations. Acta Anaesthesiol Scand 59(10):1212-1231

19. Knott A, Pathak S, McGrath JS et al (2012) Consensus views on implementation and measurement of enhanced recovery after surgery in England: Delphi study. BMJ Open 2(6):e001878 